\title{
A UNIVERSAL MULTI-COEFFICIENT THEOREM FOR THE KASPAROV GROUPS
}

\author{
Marius Dadarlat*and Terry A. LORING**
}

1. Introduction. Let $\underline{K}(A)$ denote the sum of all the $K$-theory groups of a $\mathrm{C}^{*}$-algebra $A$ in all degrees and with all cyclic coefficient groups. The Bockstein operations (which generate a category $\Lambda$ ) act on $\underline{K}(A)$. We establish a universal coefficient exact sequence

$$
0 \rightarrow \operatorname{Pext}\left(K_{*}(A), K_{*}(B)\right) \stackrel{\delta}{\rightarrow} K K(A, B) \stackrel{\Gamma}{\rightarrow} \operatorname{Hom}_{\Lambda}(\underline{K}(A), \underline{K}(B)) \rightarrow 0 .
$$

that holds in the same generality as the universal coefficient theorem of Rosenberg and Schochet.

There are advantages, in some circumstances, to using $\operatorname{Hom}_{\Lambda}(\underline{K}(A), \underline{K}(B))$ in place of $K K(A, B)$. These advantages derive from the fact that $\underline{K}(A)$ can be equipped with order and scale structures similar to those on $K_{0}(A)$. With this additional structure, the " $\Lambda$ - module" $\underline{K}(A)$ becomes a powerful invariant of $\mathrm{C}^{*}$ algebras. We show that it is a complete invariant for the class of real-rank-zero $A D$ algebras. The $A D$ algebras are a certain kind of approximately subhomogeneous $C^{*}$-algebras which may have torsion in $K_{1}$ [Ell]. In addition to classifying these algebras, we calculate their automorphism groups up to approximately innerautomorphisms.

1.1 Summary The universal coefficient theorem (UCT) of Rosenberg and Schochet [RS] states the existence of an exact sequence

$$
0 \rightarrow \operatorname{Ext}_{\mathbb{Z}}^{1}\left(K_{*}(A), K_{*}(B)\right) \stackrel{\delta}{\rightarrow} K K_{*}(A, B) \stackrel{\gamma}{\rightarrow} \operatorname{Hom}\left(K_{*}(A), K_{*}(B)\right) \rightarrow 0
$$

for many pairs of $C^{*}$-algebras $A$ and $B$. Loosely speaking, this shows that $K K_{*}(A, B)$ can be regarded as the K-theory of $B$ with coefficients in $K^{*}(A)$. In particular it shows that the coefficient group $\mathbb{Z}$ is universal in the sense that, knowing $K_{*}(B ; \mathbb{Z})=K_{*}(B)$, we can determine the $K$-theory for $B$ with other coefficients. For example,

$$
K_{*}(B ; \mathbb{Z} / p)=K K_{*}\left(A_{p}, B\right)
$$

where $A_{p}$ is an appropriate $C^{*}$-algebra with $K_{1}\left(A_{p}\right)=\mathbb{Z} / p$ and $K_{0}\left(A_{p}\right)=0$ (or $K^{0}\left(A_{p}\right)=\mathbb{Z} / p$ and $\left.K^{1}\left(A_{p}\right)=0\right)$. Mod-p K-theory for $\mathrm{C}^{*}$-algebras was considered in $[\mathrm{Kar}],[\mathrm{Cu}]$ and $[\mathrm{Sc} 1-2]$.

* partially supported by NSF grant DMS-9303361

**partially supported by NSF grant DMS-9215024 
We have recently discovered that mod- $p K$-theory can be given a very useful order structure [DL3]. More specifically, there is a order structure on

$$
K_{0}(B) \oplus K_{1}(B) \oplus K_{0}(B ; \mathbb{Z} / p) \oplus K_{1}(B ; \mathbb{Z} / p)
$$

and this order is not determined by the order on

$$
K_{0}\left(C\left(S^{1}\right) \otimes B\right)=K_{0}(B) \oplus K_{1}(B),
$$

which was defined in [DN] and [Ell]. This order is critical in the classification of $\mathrm{AD}$ (approximately dimension-drop) algebras ([Ell], [DL3], [Ei]).

The AD algebras are inductive limits of matrices over dimension-drop algebras $\tilde{\mathbb{I}}_{n}$ (see 1.2.) and over $C\left(\mathbb{T}\right.$ ). These $\mathrm{C}^{*}$-algebras may have torsion in $K_{1}$. The classification of simple AD algebras is due essentially to Elliott [Ell], but his techniques failed for nonsimple algebras as his invariant could not encode the possible complexity of the ideal structure. In order to overcome this difficulty we introduced an ordered structure on the mod- $p K$-groups. In [DL3] we used this additional structure to prove an isomorphism theorem for nonsimple AD algebras. This isomorphism result was phrased in terms of the $K K$-classes and raised the question: Given group homomorphisms (positive or not)

$$
\phi_{n}^{i}: K_{i}(A ; \mathbb{Z} / n) \rightarrow K_{i}(A ; \mathbb{Z} / n)
$$

for all $i$ and all $n$, how is it determined if there is a single $K K$-element inducing all the $\phi_{n}^{i}$ ?

To answer this, we develop a variation of the universal coefficient theorem which we call the Universal Multi-Coefficient Theorem, or the UMCT. This was in part inspired by the work of Eilers [Ei] in the context of $\mathrm{AD}$ algebras, but is stated and proven in a general setting. Applications to AD algebras will be discussed in the last section.

We define

$$
\underline{K}(A)=\bigoplus_{n=0}^{\infty} K_{*}(A ; \mathbb{Z} / n)
$$

where

$$
K_{*}(A ; \mathbb{Z} / n)=K_{0}(A ; \mathbb{Z} / n) \oplus K_{1}(A ; \mathbb{Z} / n) .
$$

By $\operatorname{Hom}_{\Lambda}(\underline{K}(A), \underline{K}(B))$ we mean all homomorphisms that respect the direct sum decomposition and the action of certain natural transformations. Some of these transformations have degree zero and are induced by coefficient morphisms $\mathbb{Z} / n \rightarrow$ $\mathbb{Z} / m$. The others have degree one and are known as the Bockstein operations. Abusing the terminology, we will call these transformations will be called the Bockstein operations. Taken together, the Bockstein operations (as defined in the $C^{*}$-algebra context by Schochet [Sc2]) form a category $\Lambda$ that acts on $\underline{K}(A)$. Rosenberg and Schochet used the UCT to study homology operations on mod-p K-theory [RS].

For groups $G$ and $H$ we define $\operatorname{Pext}(G, H)$ to be the subgroup of pure extensions in $\operatorname{Ext}(G, H)$. Recall that an extension of groups is pure if every element of finite order in the quotient lifts to an element of the same order $[\mathrm{F}]$.

The UMCT exact sequence, which is valid when $A$ is in the so-called bootstrap category of [RS] and B is $\sigma$-unital, is

$$
0 \rightarrow \operatorname{Pext}\left(K_{*}(A), K_{*}(B)\right) \stackrel{\delta}{\rightarrow} K K(A, B) \stackrel{\Gamma}{\rightarrow} \operatorname{Hom}_{\Lambda}(\underline{K}(A), \underline{K}(B)) \rightarrow 0 .
$$


As a special case, when $A$ has finitely generated $K$-theory, $\Gamma$ is an isomorphism. Several special cases of the UMCT, specifically where $A$ and $B$ are so-called ADalgebras with some restrictions on their $K$-theory, were discovered by Eilers [Ei]. Our proof of the UMCT relies heavily on the techniques used by Rosenberg and Schochet [RS] to prove the universal coefficient theorem.

The $\Lambda$-module $K(A)$, together with its order structure defines an effective invariant of $\mathrm{C}^{*}$-algebras. The UMCT allows us to define positivity for KK-elements. With these tools at hand we complete the classification started in [Ell] and [DL3] of (non-simple) AD algebras of real rank zero and compute their automorphism group modulo the approximately-inner automorphisms.

The first-named author thanks Claude Schochet and Jim McClure for useful discussions. We are grateful to Pierre de la Harpe for the references [Kar] and [B] and to Claude Schochet for making available to us early drafts of his papers [Sc3-4].

1.2. Bockstein Operations. The standard definition of mod- $p K$-theory for $C^{*}$-algebras, as given by Schochet in [Sc2], is

$$
K_{i}(A ; \mathbb{Z} / n)=K_{i}\left(C_{n} \otimes A\right)
$$

where $C_{n}$ is a commutative $C^{*}$-algebra, described below. Schochet also defines the Bockstein operations, which are natural transformations $K_{i}(-; G) \rightarrow K_{j}(-; H)$ for various indices and coefficient groups. Then he establishes exact sequences connecting these groups.

The definition of $C_{n}$ is that it is the mapping cone of a degree $n$ map of $C_{0}(\mathbb{R})$ to itself. This is the lowest-dimensional way to introduce torsion in the commutative situation. In the non-commutative setting, we may get torsion a dimension lower.

Let $\mathbb{I}_{n}$ denote the mapping cone of the unital map $\mathbb{C} \rightarrow M_{n}$, or

$$
\mathbb{I}_{n}=\left\{f \in C\left([0,1], M_{n}\right) \mid f(0)=0, f(1) \in \mathbb{C} I\right\} .
$$

This has the same $K$-theory as $C_{n}$, (or $K$-homology, as was computed in [PS]). Moreover, it is relatively easy to establish a natural isomorphism

$$
K K\left(\mathbb{I}_{n} ; A\right)=K_{0}\left(C_{n} \otimes A\right) .
$$

However, it is laborious to figure out the effect this has on the Bockstein operations. We therefore take as a definition

$$
K_{i}(A ; \mathbb{Z} / n)=K K\left(\mathbb{I}_{n}, S^{i} A\right),
$$

redefine the Bockstein operations and rederive basic facts about them.

The advantages to using $\mathbb{I}_{n}$ in the definition of mod- $p K$-theory stem from the semiprojectivity of $\mathbb{I}_{n}$ and from our previously established isomorphism

$$
\left[\mathbb{I}_{n}, A \otimes \mathcal{K}\right] \cong K K\left(\mathbb{I}_{n}, A\right)
$$

which holds when $A$ is a $\sigma$-unital $C^{*}$-algebras. (See [DL1,Lo1,Lo2].) These facts will not be used to prove the UMCT, but do indicate that our definition is a natural one. More evidence of this is given by the crucial role $\mathbb{I}_{n}$ plays in the construction 
of $\mathrm{AD}$ algebra and by the smoothing results regarding $\mathbb{I}_{n}$ and related $C^{*}$-algebras developed in $[\mathrm{LP}]$.

There are four types of Bockstein operations, each in two degrees. The operation

$$
\rho_{n}=\rho_{n}^{0} \oplus \rho_{n}^{1}, \quad \rho_{n}^{i}: K_{i}(A) \rightarrow K_{i}(A ; \mathbb{Z} / n)
$$

is given by the Kasparov product with the element of $K K\left(\mathbb{I}_{n}, \mathbf{C}\right)$ given by the function $\delta_{1}: \mathbb{I}_{n} \rightarrow \mathbf{C}$ defined by

$$
\delta_{1}(f)=f(1)
$$

The operation

$$
\beta=\beta_{n}^{0} \oplus \beta_{n}^{1}, \quad \beta_{n}^{i}: K_{i}(A ; \mathbb{Z} / n) \rightarrow K_{i+1}(A)
$$

is given by the Kasparov product with the element of $K K^{1}\left(\mathbb{C}, \mathbb{I}_{n}\right)$ determined by the obvious inclusion

$$
S M_{n} \hookrightarrow \mathbb{I}_{n}
$$

The assignment

$$
f \mapsto\left[\begin{array}{cccc}
f & & & \\
& f & & \\
& & \ddots & \\
& & & f
\end{array}\right]
$$

determines a $*$-homomorphism from $\mathbb{I}_{n}$ to $\mathbb{I}_{m n}$. Kasparov product with this determines the operation $\kappa_{n, m n}$, where

$$
\kappa_{n, m n}^{i}: K_{i}(A ; \mathbb{Z} / m n) \rightarrow K_{i}(A ; \mathbb{Z} / n) .
$$

Finally, $\kappa_{m n, m}$, with

$$
\kappa_{m n, m}^{i}: K_{i}(A ; \mathbb{Z} / m) \rightarrow K_{i}(A ; \mathbb{Z} / m n)
$$

is given by Kasparov product with the inclusion of $\mathbb{I}_{m n}$ into $M_{n}\left(\mathbb{I}_{m}\right)$, where we make the identifications

$$
M_{n}\left(\mathbb{I}_{m}\right)=\left\{f \in C_{0}\left((0,1], M_{n} \otimes M_{m}\right) \mid f(1) \in M_{n} \otimes \mathbb{C} I\right\}
$$

and

$$
\mathbb{I}_{m n}=\left\{f \in C_{0}\left((0,1], M_{n} \otimes M_{m}\right) \mid f(1) \in \mathbb{C} I \otimes \mathbb{C} I\right\} .
$$

Since the first two Bockstein operations are induced by the maps in the exact sequence

$$
0 \rightarrow S M_{n} \rightarrow \mathbb{I}_{n} \stackrel{\delta_{1}}{\longrightarrow} \mathbb{C} \rightarrow 0
$$

the exact sequence

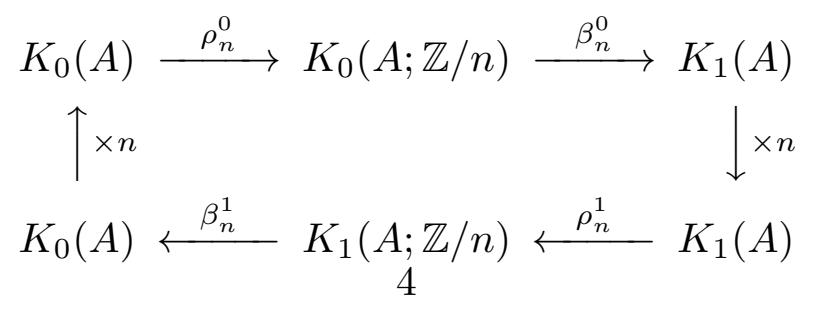


follows from the the six term exact sequence in $K K$-theory.

There is a second important exact sequence involving the Bockstein operations [Sc2]. Let us define $\beta_{m, n}^{i}=\rho_{m}^{i+1} \circ \beta_{n}^{i}$. Then this exact sequence takes the following form:

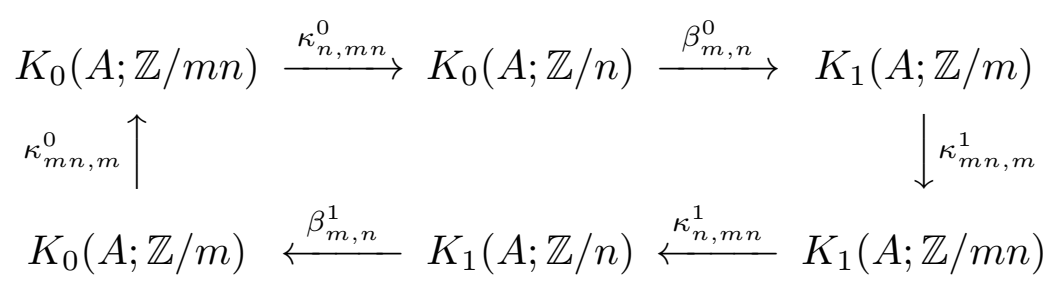

To prove this is exact we introduce an auxiliary $C^{*}$-algebra that is homotopic to $\mathbb{I}_{n}$. For any $m$, define

$$
J_{m, n}=\left\{f \in C_{0}\left((0,1]^{2}, M_{m n}\right) \mid \forall t, f(1, t) \in M_{n}, \& f(1,1) \in \mathbb{C}\right\}
$$

where we have made unital identifications

$$
\mathbb{C} \subseteq M_{n} \subseteq M_{m n}
$$

Let $\delta_{r}$ denote the evaluation map on the set $\{(1, t) \mid t \in(0,1]\}$ and let $\delta_{u}$ denote evaluation on the set $\{(s, 1) \mid s \in(0,1]\}$. It is easy to see that $\delta_{r}$ is a homotopy equivalence with $\mathbb{I}_{n}$, with the inverse $\lambda$ given by $\lambda(f)(s, t)=f(t)$.

There is a short exact sequence

$$
0 \rightarrow S M_{n}\left(\mathbb{I}_{m}\right) \stackrel{\iota}{\rightarrow} J_{m, n} \stackrel{\delta_{u}}{\rightarrow} \mathbb{I}_{m n} \rightarrow 0
$$

Which gives rise to diagram

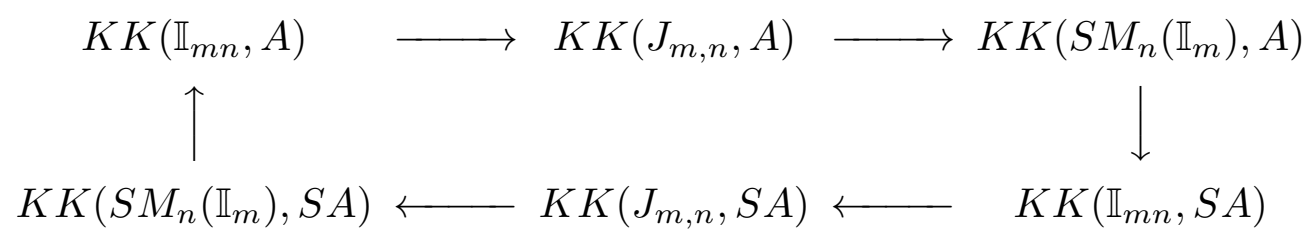

Making the identifications given by $\delta_{r}: J_{m, n} \rightarrow \mathbb{I}_{n}$, Bott periodicity and dropping matrices, have established an exact sequence of the form (1.7), but must check that the homomorphisms are the ones indicated.

It is easy to check that

$$
\delta_{u} \circ \lambda(f)=\left[\begin{array}{llll}
f & & & \\
& f & & \\
& & \ddots & \\
& & & f
\end{array}\right]
$$

and thus prove the map from $K_{0}(A ; \mathbb{Z} / m n)$ to $K_{0}(A ; \mathbb{Z} / n)$ is $\kappa_{n, m n}$.

There is also a commutative diagram with exact rows

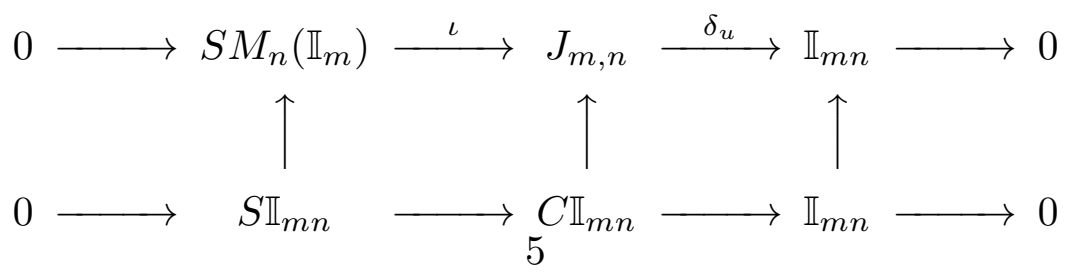


where all the vertical maps are the obvious inclusions. The bottom row, which is the usual sequence involving a cone, induces boundary map that equals [id] in $K K\left(S \mathbb{I}_{m n}, S \mathbb{I}_{m n}\right)$. It follows by naturality that the map from $K_{0}(A ; \mathbb{Z} / m)$ to $K_{0}(A ; \mathbb{Z} / m n)$ is $\kappa_{m n, m}$.

The commutative diagram

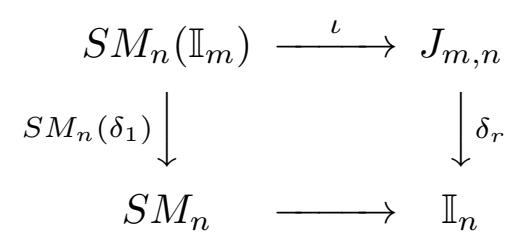

shows that the remaining two maps are $\beta_{m, n}=\rho_{m} \circ \beta_{n}$.

1.3 Examples. One obvious example to compute is $K_{*}(\mathbb{C} ; \mathbb{Z} / n)$. It follows easily from (1.2) and the definition of the $\rho$ operation that

$$
K_{0}(\mathbb{C} ; \mathbb{Z} / n)=\mathbb{Z} / n, \quad K_{1}(\mathbb{C} ; \mathbb{Z} / n)=0
$$

with $\left[\delta_{0}\right]$ the generator of $K_{0}(\mathbb{C} ; \mathbb{Z} / n)$. The composition

$$
\mathbb{I}_{n} \rightarrow \mathbb{I}_{m n}, \quad f \mapsto\left[\begin{array}{llll}
f & & & \\
& f & & \\
& & \ddots & \\
& & f
\end{array}\right]
$$

with $\delta_{0}: \mathbb{I}_{m n} \rightarrow \mathbb{C}$ is again $\delta_{0}: \mathbb{I}_{n} \rightarrow \mathbb{C}$ which proves that

$$
\kappa_{n, m n}^{1}: K_{0}(\mathbb{C} ; \mathbb{Z} / m n) \rightarrow K_{0}(\mathbb{C} ; \mathbb{Z} / n)
$$

is the map

$$
\mathbb{Z} / m n \rightarrow \mathbb{Z} / n
$$

sending [1] to [1].

Next we compute the same operation for $\mathbb{I}_{k}$. The Bockstein operations are natural and commute with suspensions (since they are given by KK-products) so from the short exact sequence

$$
0 \rightarrow S M_{k} \rightarrow \mathbb{I}_{k} \rightarrow \mathbb{C} \rightarrow 0
$$

we get a commutative diagram with exact rows:

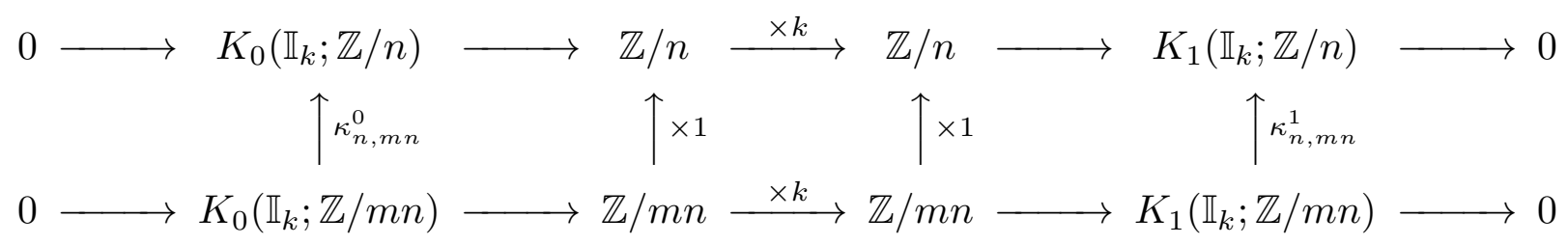

That the connecting maps are given as multiplication by $k$ can be deduced from the fact that $(1.8)$ represents the $k$ times the generator of $K K(\mathbb{C}, \mathbb{C})$. From this diagram we may conclude that

$$
K_{0}\left(\mathbb{I}_{k} ; \mathbb{Z} / n\right) \cong \mathbb{Z} /(n, k), \quad K_{1}\left(\mathbb{I}_{k} ; \mathbb{Z} / n\right) \cong \mathbb{Z} /(n, k)
$$


and that $\kappa_{n, m n}^{0}: \mathbb{Z} /(m n, k) \rightarrow \mathbb{Z} /(n, k)$ is given by

$$
\kappa_{n, m n}^{0}(\overline{1})=\frac{m(n, k)}{(m n, k)} \overline{1}
$$

and finally $\kappa_{n, m n}^{1}: \mathbb{Z} /(m n, k) \rightarrow \mathbb{Z} /(n, k)$ is given by

$$
\kappa_{n, m n}^{1}(\overline{1})=\overline{1} .
$$

1.4 The universal multi-coefficient theorem Let $\Lambda$ denote the category of Bockstein operations. The Kasparov product

$$
K K\left(\mathbb{I}_{n}, A\right) \times K K(A, B) \rightarrow K K\left(\mathbb{I}_{n}, B\right)
$$

induces a map

$$
\gamma_{n}^{0}: K K(A, B) \rightarrow \operatorname{Hom}\left(K_{0}(A ; \mathbb{Z} / n), K_{0}(B ; \mathbb{Z} / n)\right) .
$$

Similarly there is a map

$$
\gamma_{n}^{1}: K K(A, B) \rightarrow \operatorname{Hom}\left(K_{1}(A ; \mathbb{Z} / n), K_{1}(B ; \mathbb{Z} / n)\right) .
$$

Then $\gamma_{n}=\left(\gamma_{n}^{0}, \gamma_{n}^{1}\right)$ will be a map

$$
\gamma_{n}: K K(A, B) \rightarrow \operatorname{Hom}\left(K_{*}(A ; \mathbb{Z} / n), K_{*}(B ; \mathbb{Z} / n)\right) .
$$

Note that if $n=0$ then $K_{*}(A, \mathbb{Z} / n)=K_{*}(A)$ and the map $\gamma_{0}$ is the same as the map $\gamma$ from the UCT. If $n=1$ then $K_{*}(A, \mathbb{Z} / n)=0$.

For a $\sigma$-unital $\mathrm{C}^{*}$-algebra $A$ we define the group

$$
\underline{K}(A)=\bigoplus_{n=0}^{\infty} K_{*}(A ; \mathbb{Z} / n)
$$

It is a $\mathbb{Z} / 2 \times \mathbb{N}$ graded group. Via the Bockstein operations, $\underline{K}(A)$ becomes a $\Lambda$-module. It is natural to consider the group $\operatorname{Hom}_{\Lambda}(\underline{K}(A), \underline{K}(B))$ consisting of all $\mathbb{Z} / 2 \times \mathbb{N}$ graded group morphisms which are $\Lambda$-linear, i.e. preserve the action of the category $\Lambda$. Equivalently $\operatorname{Hom}_{\Lambda}(\underline{K}(A), \underline{K}(B))$ consists of sequences $\left(\phi_{n}\right)$ of $\mathbb{Z} / 2$-graded morphisms of groups

$$
\phi_{n}=\left(\phi_{n}^{0}, \phi_{n}^{1}\right): K_{*}(A ; \mathbb{Z} / n) \rightarrow K_{*}(B ; \mathbb{Z} / n)
$$

which preserve the following exact sequences

$$
K_{i}(-) \stackrel{\times n}{\longrightarrow} K_{i}(-) \stackrel{\rho_{n}^{i}}{\longrightarrow} K_{i}(-; \mathbb{Z} / n) \stackrel{\beta_{n}^{i}}{\longrightarrow} K_{i+1}(-)
$$

and

$$
K_{i+1}(-; \mathbb{Z} / n) \stackrel{\beta_{m, n}^{i+1}}{\longrightarrow} K_{i}(-; \mathbb{Z} / m) \stackrel{\kappa_{m n, m}^{i}}{\longrightarrow} K_{i}(-; \mathbb{Z} / m n) \stackrel{\kappa_{n, m n}^{i}}{\longrightarrow} K_{i}(-; \mathbb{Z} / n)
$$

for all $m, n \in \mathbb{N}, i \in \mathbb{Z} / 2$. We assemble the sequence $\left(\gamma_{n}\right)$ into a map $\Gamma$. Since the Bockstein operations are induced by multiplication with suitable KK elements and since the Kasparov product is associative, we obtain a map

$$
\Gamma: K K(A, B) \rightarrow \operatorname{Hom}_{\Lambda}(\underline{K}(A), \underline{K}(B)) .
$$

Our main result is the following universal coefficient theorem. Denote by $\mathcal{N}$ the "bootstrap" category of Rosenberg and Schochet [RS]. 
Theorem. (UMCT). Let $A, B$ be $C^{*}$-algebras. Suppose that $A \in \mathcal{N}$ and $B$ is $\sigma$-unital. Then there is a short exact sequence

$$
0 \rightarrow \operatorname{Pext}\left(K_{*}(A), K_{*}(B)\right) \stackrel{\delta}{\rightarrow} K K(A, B) \stackrel{\Gamma}{\rightarrow} H_{o m}(\underline{K}(A), \underline{K}(B)) \rightarrow 0
$$

which is natural in each variable.

Here Pext denotes the subgroup of Ext $t_{\mathbb{Z}}^{1}$ consisting of classes of pure extensions $[\mathrm{F}]$. The map $\delta$ is the restriction of the map

$$
\operatorname{Ext}_{\mathbb{Z}}^{1}\left(K_{*}(A), K_{*}(B)\right) \stackrel{\delta}{\rightarrow} K K(A, B)
$$

from the universal coefficient theorem UCT of [RS]. If $A=B$ then $\Gamma$ is a ring morphism. The proof of the UMCT uses techniques from [RS] and is based on the UCT. To identify the kernel of $\Gamma$ we use certain recent results of Schochet [Sc3-4]. The group of pure extensions is relevant for questions related to quasidiagonality and approximately unitary equivalence of $*$-homomorphisms [Br2], [KS], [Sa], $[\mathrm{BrD}],[\mathrm{R} \varnothing],[\mathrm{D}]$. The theorem is a consequence of Propositions 2.3 and 2.7 proved in the next section. By replacing $A$ by its suspension $S A$ in the UMCT and using the Bott periodicity one obtains the odd-dimensional version of the UMCT.

\section{The proof of the UMCT}

Proposition 2.1. Let $A_{1}, A_{2} \in \mathcal{N}$ and let be $B$ a $\sigma$-unital $C^{*}$-algebra. Suppose that the UMCT holds for the pair $\left(A_{1}, B\right)$. If $A_{1}$ is $K K$-equivalent to $A_{2}$, then the theorem also holds for $\left(A_{2}, B\right)$.

Proof. This very similar to the proof of Proposition 7.1 of [RS] due to Cuntz. Given an invertible element $\alpha \in K K\left(A_{2}, A_{1}\right)$, the Kasparov product with $\alpha$ induces a commutative diagram

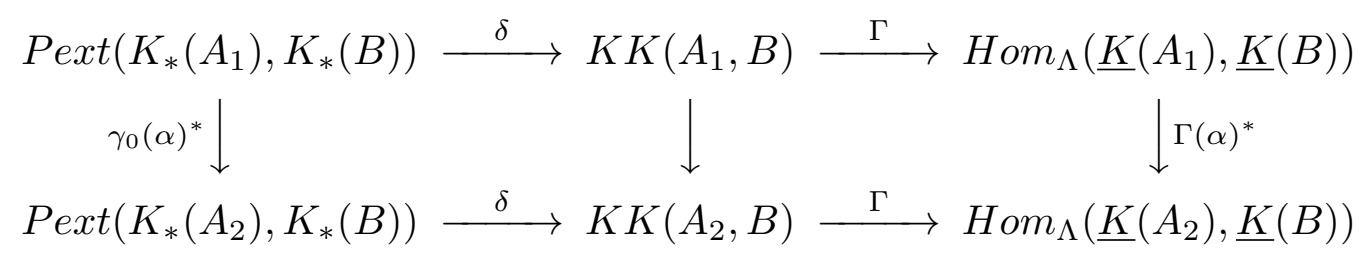

with all vertical arrows isomorphisms. The naturality of the UCT is used here. The pullback of a pure extension is pure.

Lemma 2.2. Suppose $A$ is the inductive limit of a sequence $A_{1} \rightarrow A_{2} \rightarrow \ldots$ of separable $C^{*}$-algebras. Then

(i) $\underline{K}(A)=\underline{\lim } \underline{K}\left(A_{j}\right)$ as $\Lambda$ modules.

(ii) $\operatorname{Hom}_{\Lambda}(\underline{K}(A), \underline{K}(B))=\underline{\underline{\lim }} \operatorname{Hom}_{\Lambda}\left(\underline{K}\left(A_{j}\right), \underline{K}(B)\right)$.

Proof. We omit the elementary proof based on the continuity of K-theory and the naturality of the Bockstein operations.

If $K_{*}(A)$ is finitely generated, then any pure extension of $K_{*}(A)$ is trivial and the UMCT reduces to the assertion that $\Gamma$ is an isomorphism. 
Proposition 2.3. Let $B$ be a $\sigma$-unital $C^{*}$-algebra. Suppose that the UMCT holds for the pair $(A, B)$ whenever $K_{*}(A)$ is finitely generated. Then the theorem holds for arbitrary $A \in \mathcal{N}$.

Proof. Let $A \in \mathcal{N}$. Since $A$ is separable, $K_{*}(A)$ is a countable group. Using Proposition 7.3 of [RS], we find a $\mathrm{C}^{*}$-algebra $D \in \mathcal{N}$ that is the inductive limit of a sequence $A_{1} \rightarrow A_{2} \rightarrow \ldots$ with $K_{*}\left(A_{j}\right)$ finitely generated for all $j$, and such that $D$ is KK-equivalent to $A$. By Proposition 2.1 we may assume that $A$ itself is the inductive limit of the $\left(A_{j}\right)$. Consider the following commutative diagram

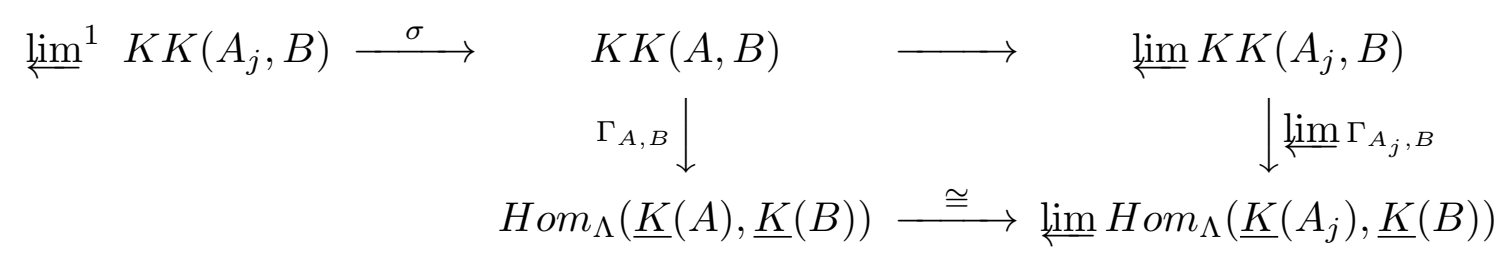

where the upper row is the Milnor $\varliminf^{1}$ exact sequence [Br1], [Sc1, Thm.7.1]. The maps $\Gamma_{A_{j}, B}$ are bijections by hypothesis. It follows that $\Gamma_{A, B}$ is surjective with kernel $\varliminf^{1} K K\left(A_{j}, B\right)$. By Theorem 4 in [Sc3], there is an exact sequence

$$
0 \rightarrow \varliminf_{\lim ^{1}} K K\left(A_{j}, B\right) \stackrel{\psi}{\rightarrow} \operatorname{Ext}_{\mathbb{Z}}^{1}\left(K_{*}(A), K_{*}(B)\right) \stackrel{\tau}{\rightarrow} \varliminf_{\lim } \operatorname{Ext}_{\mathbb{Z}}^{1}\left(K_{*}\left(A_{j}\right), K_{*}(B)\right) \rightarrow 0
$$

and a commutative diagram

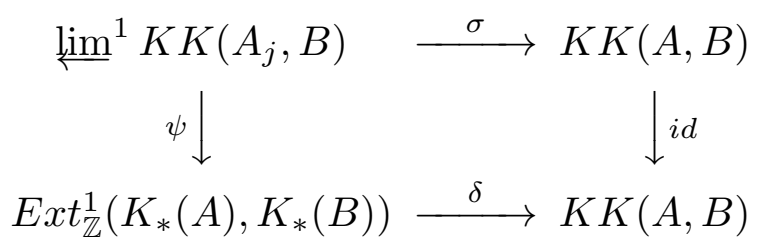

Since all $K_{*}\left(A_{j}\right)$ are finitely generated and $K_{*}(A)=\varliminf K_{*}\left(A_{j}\right)$, the kernel of $\tau$ and hence the image of $\psi$ is exactly $\operatorname{Pext}\left(K_{*}(A), K_{*}(B)\right)$. This was explain in the proof of Theorem 4.5 in [Sc4]. The general fact used here is that if $G=\underline{\varliminf} G_{j}$ where $G_{j}$ are finitely generated abelian groups, then an extension $x \in \operatorname{Ext}(G, H)$ is pure if and only if all its restrictions $x_{j} \in \operatorname{Ext}\left(G_{j}, H\right)$ are trivial. Let $\psi^{\prime}$ denote inverse of the corestriction of $\psi$ to its image. By the commutative diagram from above it is then clear that $\sigma \psi^{\prime}$ is given by the restriction of $\delta$ to $\operatorname{Pext}\left(K_{*}(A), K_{*}(B)\right)$. The naturality of $\Gamma$ is a consequence of the associativity of the Kasparov product. The naturality of $\delta$ is proved in [RS].

Proposition 2.4. Let $A$ and $B$ be as in the statement of the UMCT. Suppose that $K_{*}(A)$ is a finitely generated free group. Then $\Gamma$ is an isomorphism.

Proof. Consider the commutative diagram

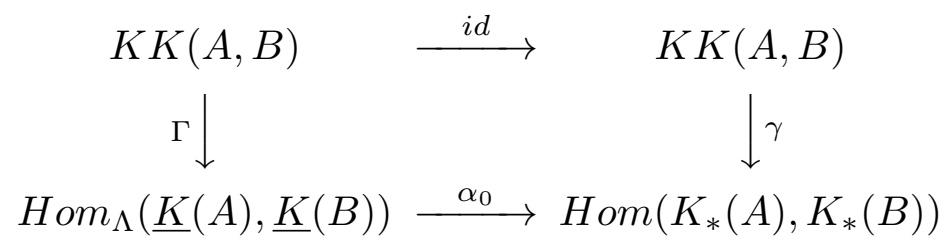

where 


$$
\alpha_{0}\left(\left(\phi_{n}\right)\right)=\phi_{0}=\left(\phi_{0}^{i}, \phi_{0}^{i+1}\right) .
$$

By the UCT, $\gamma$ is an isomorphism, hence $\alpha_{0}$ is surjective. It remains to prove that $\alpha_{0}$ is injective. Suppose that $\left(\phi_{n}\right) \in \operatorname{Hom}_{\Lambda}(\underline{K}(A), \underline{K}(B))$ is such that $\phi_{0}=0$. We are going to show that $\phi_{n}=0$ for all $\mathrm{n}$. Consider the following commutative diagram with exact rows.

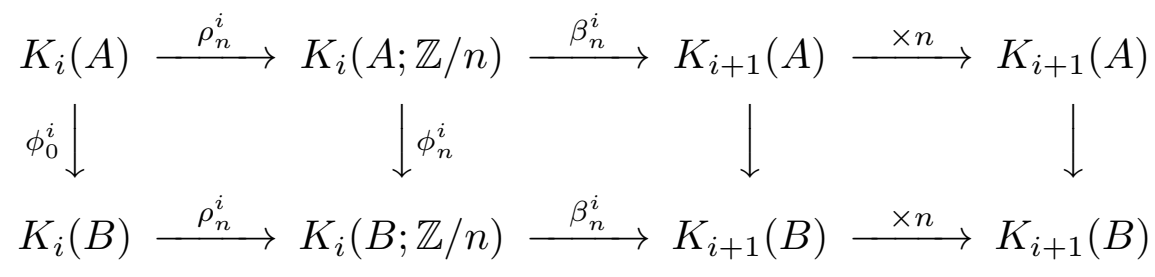

Since $K_{i+1}(A)$ is free, the multiplication by $n$ is an injective map. Thus $\beta_{n}^{i}=0$ and hence $\rho_{n}^{i}$ is surjective. Therefore from the equation $\phi_{n}^{i} \rho_{n}^{i}=\rho_{n}^{i} \phi_{0}^{i}=0$ we can conclude that $\phi_{n}^{i}=0$.

The next two Lemmas are direct consequences of the examples computed in 1.3.

Lemma 2.5. Let $A$ be a $C^{*}$-algebra in $\mathcal{N}$ with $K_{i}(A)=\mathbb{Z} / k$ and $K_{i+1}(A)=0$. Consider the Bockstein operation

$$
\kappa_{n, m n}^{i+1}: K_{i+1}(A ; \mathbb{Z} / m n) \rightarrow K_{i+1}(A ; \mathbb{Z} / n)
$$

where $m, n, k \geq 2$.

(i) If $m$ is divisible by $k$ then $\kappa_{n, m n}^{i+1}=0$.

(ii) If $k$ is divisible by $(m, k)(n, k)$ then $\kappa_{n, m n}^{i+1}$ is surjective.

Proof. By 1.3. $\kappa_{n, m n}^{i+1}: \mathbb{Z} /(m n, k) \rightarrow \mathbb{Z} /(n, k)$ is given by

$$
\kappa_{n, m n}^{i+1}(\overline{1})=\frac{m(n, k)}{(m n, k)} \overline{1}
$$

The conclusions of the Lemma follow immediately.

Lemma 2.6. Let $A$ be a $C^{*}$-algebra in $\mathcal{N}$ with $K_{i}(A)=\mathbb{Z} / k$ and $K_{i+1}(A)=0$. Consider the Bockstein operation

$$
\kappa_{n, m n}^{i}: K_{i}(A ; \mathbb{Z} / m n) \rightarrow K_{i}(A ; \mathbb{Z} / n)
$$

where $m, n, k \geq 2$.

(i) $\kappa_{n, m n}^{i}$ is surjective.

(ii) If $(m n, k)=(n, k)$ then $\kappa_{n, m n}^{i}$ is injective.

Proof. By 1.3. $\kappa_{n, m n}^{i}: \mathbb{Z} /(m n, k) \rightarrow \mathbb{Z} /(n, k)$ is given by $\kappa_{n, m n}^{i}(\overline{1})=\overline{1}$. The conclusions of the Lemma are now obvious. 
Proposition 2.7. Let $A, B$ be $C^{*}$-algebras. Suppose that $A \in \mathcal{N}$ and $B$ is $\sigma$ unital. Suppose that $K_{i}(A)=\mathbb{Z} / k$ and $K_{i+1}(A)=0$ where $k=p^{a}$, $p$ is a prime and $a \geq 1$. Then the restriction map

$$
\operatorname{Hom}_{\Lambda}(\underline{K}(A), \underline{K}(B)) \stackrel{\alpha_{k}^{i+1}}{\longrightarrow} \operatorname{Hom}\left(K_{i+1}(A ; \mathbb{Z} / k), K_{i+1}(B ; \mathbb{Z} / k)\right)
$$

is injective.

Proof. Let $\phi=\left(\left(\phi_{n}^{i}, \phi_{n}^{i+1}\right)\right) \in \operatorname{Hom}_{\Lambda}(\underline{K}(A), \underline{K}(B))$ and assume that $\phi_{k}^{i+1}=0$. We will show that this implies that $\phi=0$. For the first two parts of the proof our arguments rely on the following commutative diagrams with exact rows.

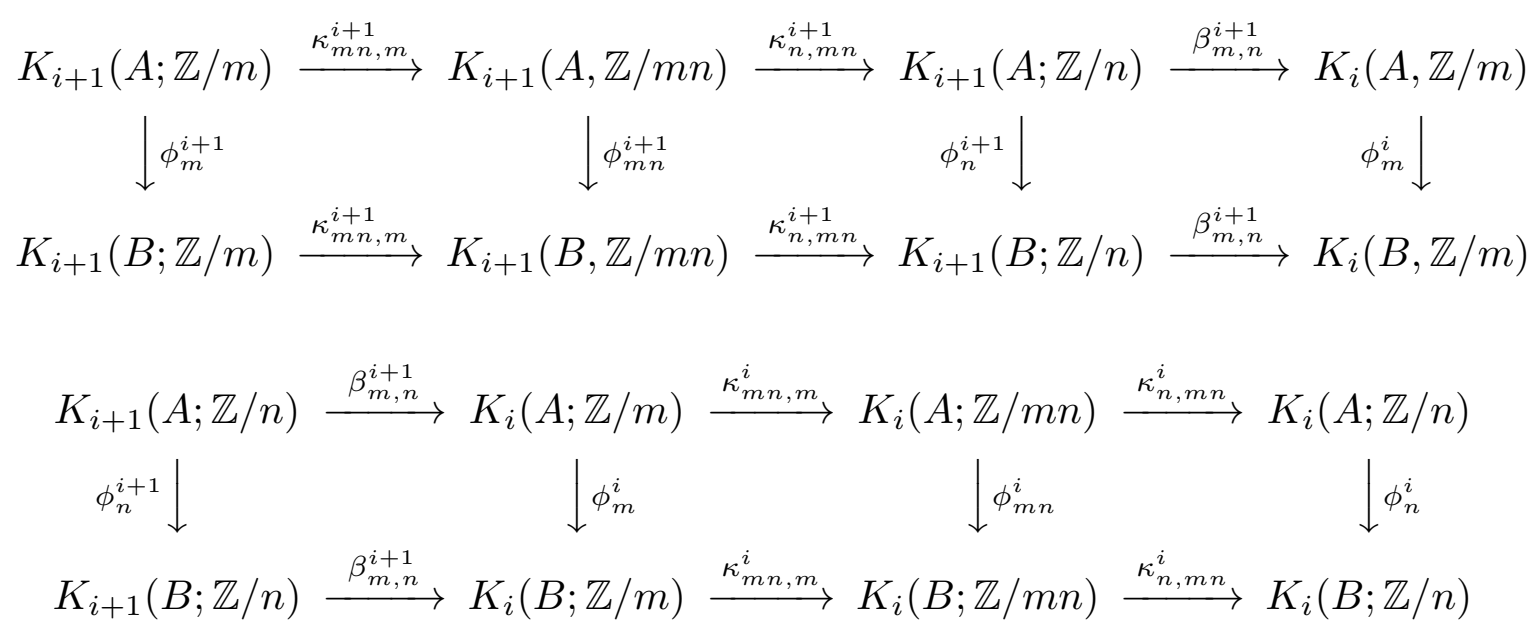

We show first that $\phi_{r}^{i+1}=0$ for all $r \geq 2$. Write $r=p^{b} s$ with $(s, p)=1$. If $b \geq a$, set $m=k=p^{a}$ and $n=p^{b-a} s$. Since $m$ is divisible by $k$, Lemma 2.5 (i) shows that $\kappa_{n, m n}^{i+1}=0$ and therefore $\kappa_{m n, m}^{i+1}$ is surjective. Since $\phi_{m}^{i+1}=\phi_{k}^{i+1}=0$, $\phi_{m n}^{i+1} \kappa_{m n, m}^{i+1}=\kappa_{m n, m}^{i+1} \phi_{m}^{i+1}=0$ hence $\phi_{m n}^{i+1}=\phi_{r}^{i+1}=0$. If $b \leq a$, set $n=r=p^{b} s$ and $m=p^{a-b}$. Then $(m, k)(n, k)=p^{a-b} p^{b}=p^{a}$. By Lemma 2.5 (ii), it follows that $\kappa_{n, m n}^{i+1}$ is surjective. Since $\phi_{n}^{i+1} \kappa_{n, m n}^{i+1}=\kappa_{n, m n}^{i+1} \phi_{m n}^{i+1}=0$, this implies that $\phi_{n}^{i+1}=\phi_{r}^{i+1}=0$.

In the second part of the proof we show that that $\phi_{r}^{i}=0$ for all $r \geq 2$. Set $m=r$ and $n=k$. Then $(m n, k)=k=(n, k)$, hence $\kappa_{n, m n}^{i}$ is injective by Lemma 2.6 (ii). By the exactness of the upper row of the second diagram, this implies that $\kappa_{m n, m}^{i}=0$. Therefore $\beta_{m, n}^{i+1}$ must be surjective. Since $\phi_{n}^{i+1}=0$ by the first part of the proof, $\phi_{m}^{i} \beta_{m, n}^{i+1}=\beta_{m, n}^{i+1} \phi_{n}^{i+1}=0$. We conclude that $\phi_{r}^{i}=\phi_{m}^{i}=0$.

In the last part of the proof we show that $\phi_{0}^{0}=\phi_{0}^{1}=0$. It is clear that $\phi_{0}^{i+1}=0$ since $K_{i+1}(A)=0$. From the commutative diagram

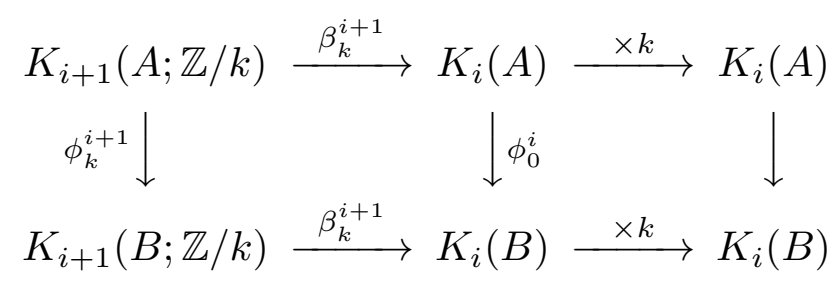

it follows that $\phi_{0}^{i} \beta_{k}^{i+1}=\beta_{k}^{i+1} \phi_{k}^{i+1}=0$ since we have already seen that $\phi_{k}^{i+1}=0$. The map $\beta_{k}^{i+1}$ is surjective since $k K_{i}(A)=0$. We conclude that $\phi_{0}^{i}=0$. 
Proposition 2.8. Let $A_{1}, A_{2} \in \mathcal{N}$ and let $B$ be a $\sigma$-unital $C^{*}$-algebra. Suppose that $\Gamma_{A_{1}, B}$ and $\Gamma_{A_{2}, B}$ are isomorphisms. Then $\Gamma_{A_{1} \oplus A_{2}, B}$ is an isomorphism.

Proof. We leave the proof for the reader. One uses the fact that both $K K(A, B)$ and $\operatorname{Hom}_{\Lambda}(\underline{K}(A), \underline{K}(B))$ are additive in the first variable.

Proposition 2.9. Let $A \in \mathcal{N}$ and let $B$ be a $\sigma$-unital $C^{*}$-algebra. Suppose that $K_{*}(A)$ is finitely generated. Then the map

$$
\Gamma: K K(A, B) \rightarrow \operatorname{Hom}_{\Lambda}(\underline{K}(A), \underline{K}(B))
$$

is an isomorphism.

Proof. By Proposition 2.1 and Proposition 7.3 of [RS] we may assume that $A$ is isomorphic to a finite sum of $\mathrm{C}^{*}$-algebras $A_{j}$ such that each $K_{*}\left(A_{j}\right)$ is a primary cyclic group supported in one dimension or isomorphic to $\mathbb{Z}$. By Proposition 2.8 we may further assume that $A$ is equal to some $A_{j}$ and therefore it is enough to consider the case when $K_{i+1}(A)=0$ and $K_{i}(A)=\mathbb{Z}$ or $K_{i}(A)=\mathbb{Z} / p^{a}$ for some prime $p$ and $a \geq 1$. The torsion free case was settled in Proposition 2.4. Therefore we only need to prove the statement in the case when $K_{i+1}(A)=0$ and $K_{i}(A)=\mathbb{Z} / p^{a}$. Set $k=p^{a}$. By Proposition 2.1 we may assume that $A=S^{i+1} \mathbb{I}_{k}$. Consider the commutative diagram

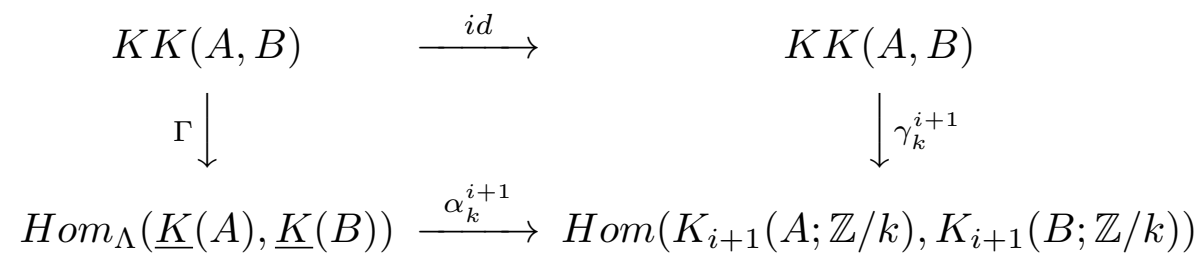

First we show that $\gamma_{k}^{i+1}$ is a bijection. Via the identifications

$$
\begin{gathered}
K_{i+1}\left(S^{i+1} \mathbb{I}_{k} ; \mathbb{Z} / k\right) \cong K K\left(\mathbb{I}_{k}, S^{2 i+2} \mathbb{I}_{k}\right) \cong K K\left(\mathbb{I}_{k}, \mathbb{I}_{k}\right) \\
K_{i+1}(B ; \mathbb{Z} / k) \cong K K\left(\mathbb{I}_{k}, S^{i+1} B\right) \cong K K\left(S^{i+1} \mathbb{I}_{k}, B\right)
\end{gathered}
$$

the map $\gamma_{k}^{i+1}$ is induced by the Kasparov product

$$
K K\left(\mathbb{I}_{k}, \mathbb{I}_{k}\right) \times K K\left(S^{i+1} \mathbb{I}_{k}, B\right) \rightarrow K K\left(S^{i+1} \mathbb{I}_{k}, B\right) .
$$

This clearly shows that $\gamma_{k}^{i+1}$ is a bijection. From the above commutative diagram, it follows that $\alpha_{k}^{i+1}$ is surjective. By Proposition 2.7, $\alpha_{k}^{i+1}$ is injective. We conclude that $\Gamma$ is bijective.

Let $P \subset \mathbb{N}$ be the set consisting of the positive powers of all the primes. We define

$$
F_{P} \underline{K}(A)=K_{*}(A) \oplus \bigoplus_{k \in P} K_{*}(A ; \mathbb{Z} / k) .
$$

There is a canonical subcategory of $\Lambda$ acting on $F_{P} \underline{K}(A)$. Similarly for any positive integer $n$ we define

$$
\begin{gathered}
F_{n} \underline{K}(A)=K_{*}(A) \oplus \bigoplus_{k \mid n} K_{*}(A ; \mathbb{Z} / k) . \\
F_{n}^{\prime} \underline{K}(A)=K_{*}(A) \oplus \bigoplus_{\substack{k \in P \\
k \mid n}} K_{*}(A ; \mathbb{Z} / k) .
\end{gathered}
$$

Again there are suitable subcategories of $\Lambda$ acting on these groups and which are maximal with respect this property. 
Proposition 2.10. Let $A \in \mathcal{N}$ and let $B$ be a $\sigma$-unital $C^{*}$-algebra. Then the natural restriction map

$$
\operatorname{Hom}_{\Lambda}(\underline{K}(A), \underline{K}(B)) \rightarrow \operatorname{Hom}_{\Lambda}\left(F_{P} \underline{K}(A), F_{P} \underline{K}(B)\right)
$$

is an isomorphism of groups.

Proof. The both groups in the statement can be regarded as contravariant functors in the $A$ variable. These functors preserve the direct sums, are continuous and behave nicely with respect KK-equivalences. The restriction map can be regarded as a natural transformation between the two functors which is compatible with the above operations. Therefore, as in the proof of Proposition 2.9, it is enough to deal with the particular case when $K_{i+1}(A)=0$ and $K_{i}(A)=\mathbb{Z}$ or $K_{i}(A)=$ $\mathbb{Z} / p^{a}$ with $p$ prime and $a \geq 0$. The statement now follows by reasoning as in the proofs of Propositions 2.4, 2.7 and 2.9 with $\operatorname{Hom}_{\Lambda}\left(F_{P} \underline{K}(A), F_{P} \underline{K}(B)\right)$ replacing $\operatorname{Hom}_{\Lambda}(\underline{K}(A), \underline{K}(B))$.

The next result is derived by similar arguments.

Corollary 2.11. Let $A \in \mathcal{N}$ and let $B$ be a $\sigma$-unital $C^{*}$-algebra. Suppose that there is a positive integer $n$ such that the torsion subgroup $T$ of $K_{*}(A)$ satisfies $n T=0$. Then the natural restriction maps induce isomorphisms

$$
\operatorname{Hom}_{\Lambda}(\underline{K}(A), \underline{K}(B)) \rightarrow \operatorname{Hom}_{\Lambda}\left(F_{n} \underline{K}(A), F_{n} \underline{K}(B)\right) \rightarrow \operatorname{Hom}_{\Lambda}\left(F_{n}^{\prime} \underline{K}(A), F_{n}^{\prime} \underline{K}(B)\right) .
$$

\section{A new invariant, applications}

All the $\mathrm{C}^{*}$-algebras in this section are assumed to be $\sigma$-unital. Throughout this section the $K K$-class of a $*$-homomorphism $\varphi: A \rightarrow B$ will be denoted by $[\varphi]$ and this should not be confused with the homotopy class of $\varphi$.

In [DL3] we introduced a preorder structure on the groups

$$
K_{0}(A ; \mathbb{Z} \oplus \mathbb{Z} / n)=K_{0}(A) \oplus K_{0}(A ; \mathbb{Z} / n)
$$

and

$$
K_{*}(A ; \mathbb{Z} \oplus \mathbb{Z} / n)=K_{*}(A) \oplus K_{*}(A ; \mathbb{Z} / n) .
$$

There are isomorphisms

$$
K_{0}(A ; \mathbb{Z} \oplus \mathbb{Z} / n) \cong K K\left(\tilde{\mathbb{I}}_{n}, A\right)
$$

and

$$
K_{*}(A ; \mathbb{Z} \oplus \mathbb{Z} / n) \cong K K\left(\tilde{\mathbb{I}}_{n}, A \otimes C(\mathbb{T})\right)
$$

induced by the split-exact sequence

$$
0 \rightarrow \mathbb{I}_{n} \rightarrow \tilde{\mathbb{I}}_{n} \rightarrow \mathbb{C} \rightarrow 0
$$

Treating these as identifications, we define the positive elements as those that are represented by $*$-homomorphisms from $\tilde{\mathbb{I}}_{n}$ to $A \otimes \mathcal{K}$, or to $A \otimes C(\mathbb{T}) \otimes \mathcal{K}$. 
These preorder structures share many of the properties held by the preorder structure on $K_{0}(A)$. Notice that

$$
K_{*}(A ; \mathbb{Z} \oplus \mathbb{Z} / n)^{+}=K_{0}(A \otimes C(\mathbb{T}) ; \mathbb{Z} \oplus \mathbb{Z} / n)^{+}
$$

under the usual identification of the underlying groups. The preoder structure on $K_{0}(A ; \mathbb{Z} \oplus \mathbb{Z} / n)$ encompasses that on $K_{0}(A)$ in the sense that if $\left(x_{0}, x_{n}\right)$ is in $K_{0}(A ; \mathbb{Z} \oplus \mathbb{Z} / n)^{+}$then $x_{0}$ is in $K_{0}(A)^{+}$.

Recall from [DL3] that

$$
\left[\mathbb{I}_{n}, A \otimes \mathcal{K}\right] \cong K K\left(\mathbb{I}_{n}, A\right)=K_{0}(A ; \mathbb{Z} / n) .
$$

The fact that $\mathbb{I}_{n}$ and $\tilde{\mathbb{I}}_{n}$ have stable relations tells us moreover that

$$
\varliminf_{\longrightarrow}\left[\mathbb{I}_{n}, M_{r}(A)\right]=\left[\mathbb{I}_{n}, A \otimes \mathcal{K}\right]=K_{0}(A ; \mathbb{Z} / n)
$$

and

$$
\varliminf_{\longrightarrow}\left[\tilde{\mathbb{I}}_{n}, M_{r}(A)\right]=\left[\tilde{\mathbb{I}}_{n}, A \otimes \mathcal{K}\right] .
$$

A more general consequence is that $\left[\mathbb{I}_{n},-\right]$ and $\left[\tilde{\mathbb{I}}_{n},-\right]$ are continuous.

In what follows a $\mathrm{C}^{*}$-algebra $A$ will be called stably unital if $A$ has an approximate unit consisting of projections. (Note that this definition is slightly more restrictive than the one given in [B11].)

Proposition 3.1. Suppose that $A$ is a stably unital $C^{*}$-algebra. Then

(i) $K K\left(\tilde{\mathbb{I}}_{n}, A\right)=\left\{[\varphi]-[\psi]: \varphi, \psi \in \operatorname{Hom}\left(\tilde{\mathbb{I}}_{n}, A \otimes \mathcal{K}\right)\right\}$

(ii) $[\varphi]=[\psi]$ if and only if there is $\eta \in \operatorname{Hom}\left(\tilde{\mathbb{I}}_{n}, A \otimes \mathcal{K}\right)$ vanishing on $\mathbb{I}_{n}$ such that $\varphi \oplus \eta$ is homotopic to $\psi \oplus \eta$. Therefore $K K\left(\tilde{\mathbb{I}}_{n}, A\right) \cong K_{0}(A ; \mathbb{Z} \oplus \mathbb{Z} / n)$ is isomorphic to the Grothendieck group of the semigroup $\left[\tilde{\mathbb{I}}_{n}, A \otimes \mathcal{K}\right]$.

Proof. We give the proof only for unital $\mathrm{C}^{*}$-algebras $A$. The general case will follow by writing $A$ as an inductive limit of unital $\mathrm{C}^{*}$-algebras and by using the continuity of $\left[\tilde{\mathbb{I}}_{n},-\right]$ and $K K\left(\tilde{\mathbb{I}}_{n},-\right)$.

(i) Essentially, this part is contained in Proposition 2.2 of [DL3]. Let $x=\left(x_{0}, x_{n}\right)$ be an element of $K_{0}(A) \oplus K K\left(\mathbb{I}_{n}, A\right)$. Let $p, q$ be projections in $M_{s}(A)$ such that $x_{0}=[p]-[q]$. For some $s$ there is an elememt $\varphi_{0}$ of $\operatorname{Hom}\left(\mathbb{I}_{n}, M_{r}(A)\right)$ with $\left[\varphi_{0}\right]=x_{n}$. Extend $\varphi_{0}$ to a $*$-homomorphism $\varphi: \tilde{\mathbb{I}}_{n} \rightarrow M_{r+s}(A)$ by setting $\varphi(1)=1_{r} \oplus p$ where $1_{r}$ denotes the unit of $M_{r}(A)$. Let $\psi: \tilde{\mathbb{I}}_{n} \rightarrow M_{r+s}$ be a $*$-homomorphism with kernel $\mathbb{I}_{n}$ and such that $\psi(1)=1_{r} \oplus q$. It is then clear that $[\varphi]-[\psi]=x$.

(ii) We begin be reducing the proof to the case when $\varphi(1)=\psi(1)$. By the discussion above we may assume that $\varphi, \psi \in \operatorname{Hom}\left(\tilde{\mathbb{I}}_{n}, M_{r}(A)\right)$ for some $r$. If $[\varphi]=$ $[\psi]$, then $[\varphi(1)]=[\psi(1)]$ in $K_{0}(A)$. It follows that there exist a projection $q \in M_{s}(A)$ and a unitary $u \in U_{r+s}(A)$ homotopic to the identity, such that

$$
u(\varphi(1) \oplus q) u^{*}=\psi(1) \oplus q .
$$

Let $\eta: \tilde{\mathbb{I}}_{n} \rightarrow M_{s}$ be a $*$-homomorphism with kernel $\mathbb{I}_{n}$ and such that $\eta(1)=q$. It is then clear that $\varphi \oplus \eta$ is homotopic to $u(\varphi \oplus \eta) u^{*}$. This shows that without any loss of generality we may assume that $\varphi(1)=\psi(1)=e \leq 1_{r}$. Since $\left[\left.\varphi\right|_{\mathbb{I}_{n}}\right]=\left[\left.\psi\right|_{\mathbb{I}_{n}}\right]$, there exist $R \geq r$ and a homotopy $\Gamma_{t}: \mathbb{I}_{n} \rightarrow M_{R}(A)$ from $\left.\varphi\right|_{\mathbb{I}_{n}}$ to $\left.\psi\right|_{\mathbb{I}_{n}}$. Extend $\Gamma_{t}$ to $\tilde{\mathbb{I}}_{n}$ by setting $\Gamma_{t}(1)=1_{R}$. It is then obvious that $\Gamma_{t}$ is a homotopy from $\varphi \oplus \eta_{1}$ to $\psi \oplus \eta_{1}$, where $\eta_{1}$ is a $*$-homomorphism vanishing on $\mathbb{I}_{n}$ with $\eta_{1}(1)=1_{R}-e$. 
Proposition 3.2. Suppose that $A$ is a stably unital, stably finite $C^{*}$-algebra. Then

$$
\left(K_{0}(A ; \mathbb{Z} \oplus \mathbb{Z} / n), K_{0}(A ; \mathbb{Z} \oplus \mathbb{Z} / n)^{+}\right)
$$

is an ordered group. Moreover if $\left(x_{0}, x_{n}\right)$ is in $K_{0}(A ; \mathbb{Z} \oplus \mathbb{Z} / n)^{+}$and $x_{0}=0$, then $x_{n}=0$.

Proof. If $G=K_{0}(A ; \mathbb{Z} \oplus \mathbb{Z} / n)$, then $G^{+}-G^{+}=G$ by Proposition 3.1 (i). It remains to show that $G^{+} \cap\left(-G^{+}\right)=0$. If $x \in G^{+} \cap\left(-G^{+}\right)=0$, then there exist *-homomorphisms $\varphi, \psi: \tilde{\mathbb{I}}_{n} \rightarrow M_{r}(A)$ with $x=[\varphi]=-[\psi]$, hence $[\varphi \oplus \psi]=0$ in $K K\left(\tilde{\mathbb{I}}_{n}, A\right)$. Therefore $[\varphi(1) \oplus \psi(1)]=0$ in $K_{0}(A)$. Under the same assumptions it is proved in Proposition 6.3.3 of [Bl1] that $\left(K_{0}(A), K_{0}(A)^{+}\right)$is an ordered group. It follows that $\varphi(1)=\psi(1)=0$, hence $\varphi=\psi=0$. To prove the second part of the statement, write $\left(x_{0}, x_{n}\right)=[\varphi]$ for some $*$-homomorphism $\varphi: \tilde{\mathbb{I}}_{n} \rightarrow M_{r}(A)$. If $x_{0}=[\varphi(1)]=0$, then as above $\varphi(1)=0$, hence $\varphi=0$. We conclude that $x_{n}=\left[\left.\varphi\right|_{\mathbb{I}_{n}}\right]=0$.

Under the assumptions of Proposition 3.2 it easily seen that $A \otimes C(\mathbb{T})$ is stably unital and stably finite. Therefore

$$
\left(K_{*}(A ; \mathbb{Z} \oplus \mathbb{Z} / n), K_{*}(A ; \mathbb{Z} \oplus \mathbb{Z} / n)^{+}\right)
$$

is also an ordered group.

Definition 3.3. Let $\underline{K}(A)^{+}$denote the semigroup in $\underline{K}(A)$ generated by union of $K_{*}(A ; \mathbb{Z} \oplus \mathbb{Z} / n)^{+}$taken over all $n \in \mathbb{N}$. A morphism in $\operatorname{Hom}_{\Lambda}(\underline{K}(A), \underline{K}(B))$ is called positive if it maps $\underline{K}(A)^{+}$into $\underline{K}(B)^{+}$.

Let $x$ be an element of $\underline{K}(A)$ so that $x=\left(x_{0}, x_{1}, \ldots\right)$ with $x_{0} \in K_{*}(A)$ and $x_{n} \in K_{*}(A ; \mathbb{Z} / n)$ for $n>0$. By definition, $x$ is positive if and only if there are $y_{n}$ in $K_{*}(A)$ such that $\left(y_{n}, x_{n}\right)$ is a positive element of $K_{*}(A ; \mathbb{Z} \oplus \mathbb{Z} / n)^{+}$for $n>0$, with all but finitely many equal to zero, and $x_{0}=\sum_{j>0} y_{j}$. As explained before Proposition 3.1, each $y_{k}$ must be in $K_{*}(A)^{+}$, hence $\left(x_{0}, x_{n}\right) \geq\left(y_{n}, x_{n}\right)$. This shows that if $x$ is positive, then all $\left(x_{0}, x_{n}\right)$ are positive. An obvious consequence is that a morphism $\Phi=\left(\Phi_{n}\right): \underline{K}(A) \rightarrow \underline{K}(B)$ is positive if and only if all its restrictions

$$
\left(\Phi_{0}, \Phi_{n}\right): K_{*}(A ; \mathbb{Z} \oplus \mathbb{Z} / n) \rightarrow K_{*}(B ; \mathbb{Z} \oplus \mathbb{Z} / n)
$$

are positive.

Let $G$ denote one of the groups $K_{0}(A), K_{*}(A), K_{0}(A ; \mathbb{Z} \oplus \mathbb{Z} / n)$. Then $G$ is natural direct summand of $K_{*}(A ; \mathbb{Z} \oplus \mathbb{Z} / n)$ and the projection onto $G$ is given by composition with a suitable $*$-homomorphism. This shows immediately that $K_{*}(A ; \mathbb{Z} \oplus \mathbb{Z} / n)^{+} \cap G=G^{+}$. It follows that if $\Phi$ is positive, then its restriction to $G$ is also positive.

Proposition 3.4. Suppose that $A$ is a stably unital, stably finite $C^{*}$-algebra. Then $\left(\underline{K}(A), \underline{K}(A)^{+}\right)$is an ordered group.

Proof. $\underline{K}(A)=\underline{K}(A)^{+}-\underline{K}(A)^{+}$since the corresponding property is true for each of the subgroups $K_{*}(A ; \mathbb{Z} \oplus \mathbb{Z} / n)$ and these subgroups generate $\underline{K}(A)$. It remains to prove that $\underline{K}(A)^{+} \cap\left(-\underline{K}(A)^{+}\right)=0$. To this purpose we show that if $x, y \in \underline{K}(A)^{+}$ and $x+y=0$, then $x=y=0$. Write $x=\left(x_{n}\right)$ and $y=\left(y_{n}\right)$ with $x_{n}, y_{n} \in$ 
$K_{*}(A ; \mathbb{Z} / n)$. Since both $x$ and $y$ are positive, $\left(x_{0}, x_{n}\right),\left(y_{0}, y_{n}\right) \in K_{*}(A ; \mathbb{Z} \oplus \mathbb{Z} / n)^{+}$. Since this is an ordered group and $\left(x_{0}, x_{n}\right)+\left(y_{0}, y_{n}\right)=(0,0)$, both elements must be zero, hence $x=y=0$.

Recall that the scale $\Sigma(A)$ of a $\mathrm{C}^{*}$-algebra $A$ is defined as the image of $[\mathbb{C}, A]$ in $K_{0}(A)=K K(\mathbb{C}, A)$. The scale of $A \otimes C(\mathbb{T})$ is denoted by $\Sigma_{*}(A)$. For unital $\mathrm{C}^{*}$ algebras $A$ with cancellation of projections, the scale is equal to the order interval of those $x$ in $K_{0}(A)$ such that $0 \leq x \leq[1]$ (see [Bl1]). We extend this notion by defining $\underline{\Sigma}(A)$ to be the union of the images of $\operatorname{Hom}\left(\tilde{\mathbb{I}}_{n}, A \otimes C(\mathbb{T})\right)$ in $\underline{K}(A)$.

While in general $\Sigma(A)$ is a stronger invariant than $\Sigma(A)$, for stably unital, stable finite $\mathrm{C}^{*}$-algebras $A$ such that $A \otimes C(\mathbb{T})$ has cancellation of projections, $\underline{K}(A)^{+}$and $\Sigma_{*}(A)$ do determine $\underline{\Sigma}(A)$. More precisely, if $x$ is in $K_{*}(A ; \mathbb{Z} \oplus \mathbb{Z} / n)^{+} \subset \underline{K}(A)^{+}$, then $x$ is in $\Sigma(A)$ if and only if its $K_{*}(A)$ component $x_{0}$ is in $\Sigma_{*}(A)$. Indeed, let us suppose that $x=[\varphi]$ for some $*$-homomorphism $\varphi: \tilde{\mathbb{I}}_{n} \rightarrow M_{r}(A \otimes C(\mathbb{T}))$. Then $x_{0} \in \Sigma_{*}(A)$ if and only if $[\varphi(1)]=[p]$ for some projection $p$ in $A \otimes C(\mathbb{T})$. If $A \otimes C(\mathbb{T})$ has cancellation of projections then $\varphi(1)$ and $p$ are equivalent via some partial isometry $v \in A \otimes C(\mathbb{T}) \otimes \mathcal{K}$, hence $[\varphi]=\left[v \varphi v^{*}\right] \in \Sigma(A)$. It can be shown that for many approximately subhomogeneous $\mathrm{C}^{*}$-algebras of real rank zero $\underline{K}(A)^{+}$ and $\Sigma(A)$ determine $\Sigma(A)$, but we don't need this fact here.

Definition 3.5. The $\mathbf{K}$-invariant of a $C^{*}$-algebra $A$ is the preordered scaled group $\left(\underline{K}(A), \underline{K}(A)^{+}, \Sigma(A)\right)$ together with the action of the Bockstein operations on $\underline{K}(A)$.

We define $\operatorname{Hom}_{\Lambda}(\underline{K}(A), \underline{K}(B))^{+}$to consist of all positive morphisms in $H^{\prime} m_{\Lambda}(\underline{K}(A), \underline{K}(B))$, and $K K(A, B)^{+}$to consist of all those elements $\alpha \in K K(A, B)$ such that $\Gamma(\alpha) \in \operatorname{Hom}_{\Lambda}(\underline{K}(A), \underline{K}(B))^{+}$.

Before going to applications it is worth mentioning that are alternative ways to put a preorder structure on $\underline{K}(A)$. Let $X_{n}$ be the space obtained by attaching the disk to the circle via a degree $n$ selfmap of the circle. Since $K_{*}(A ; \mathbb{Z} \oplus \mathbb{Z} / n)$ is isomorphic to the Grothendieck group of the semigroup

$$
\left[\mathbb{C}, A \otimes C(\mathbb{T}) \otimes C\left(X_{n}\right) \otimes \mathcal{K}\right]
$$

we can simply define $K_{*}(A ; \mathbb{Z} \oplus \mathbb{Z} / n)^{+}$to be the image of the semigroup in $K_{*}(A ; \mathbb{Z} \oplus$ $\mathbb{Z} / n)$. While in general different from the preorder given in Definition 3.1, this preorder carries similar information in the case of AH algebras. Naturally, the definition of the $\mathbf{K}$-invariant in this setting will use the Bockstein operations as defined in [Sc2].

Recall that an $\mathrm{AD}$ algebra is a $\mathrm{C}^{*}$-algebra isomorphic to an inductive limit of direct sums of matrices over circle algebras and dimension-drop algebras [Ell]. If $A$ is an AD algebra, then $A$ is stably unital and stably finite, hence then the $\mathbf{K}$ invariant becomes an ordered, scaled group. The next result shows that $\mathbf{K}$ is a complete invariant for the $\mathrm{AD}$ algebras of real rank zero.

Theorem 3.6. Suppose that $A$ and $B$ are $A D$ algebras of real rank zero. Suppose that there is a graded isomorphism of ordered, scaled groups $\phi: \underline{K}(A) \rightarrow \underline{K}(B)$ that commutes with the Bockstein operations. Then $\phi$ lifts to a $*$-isomorphism $\varphi: A \rightarrow B$.

Proof. Write $A=\varliminf_{\longrightarrow}\left(A_{n}, p_{m, n}\right)$ and $B=\varliminf_{(}\left(B_{n}, q_{m, n}\right)$ where $A_{n}$ and $B_{n}$ are basic blocks and $p_{m, n}, q_{m, n}$ are the connecting maps. Let $p_{n}: A_{n} \rightarrow A$ and $q_{n}: B_{n} \rightarrow B$ be the obvious mappings of $A_{n}, B_{n}$ into $A, B$. By the discussion 
following Definition 3.3, $\phi$ induces isomorphisms of ordered groups $K_{*}(A) \rightarrow K_{*}(B)$ and $K_{0}(A ; \mathbb{Z} \oplus \mathbb{Z} / n) \rightarrow K_{0}(B ; \mathbb{Z} \oplus \mathbb{Z} / n)$. Using the UMCT we can lift $\phi$ to a $\mathrm{KK}$ equivalence $\alpha \in K K(A, B)$. By Theorem 4.1 of [DL3] it follows now that there is a $*$-isomorphism $\varphi: A \rightarrow B$. It remains to show that $\Gamma([\varphi])=\phi$, where $[\varphi]$ denotes the KK class of $\varphi$. Let us recall the construction of $\varphi$. First we produced a commutative KK diagram

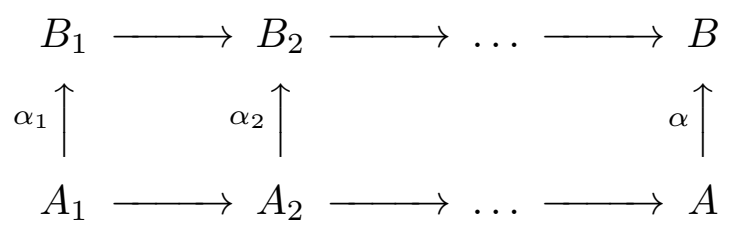

Several times during the proof we did reindex the two inductive systems. Using positivity arguments we showed that the $\alpha_{i}$ are induced by $*$-homomorphisms $\varphi_{i}$ : $A_{i} \rightarrow B_{i}$ and as in [Ell] one produces an approximately commutative diagram

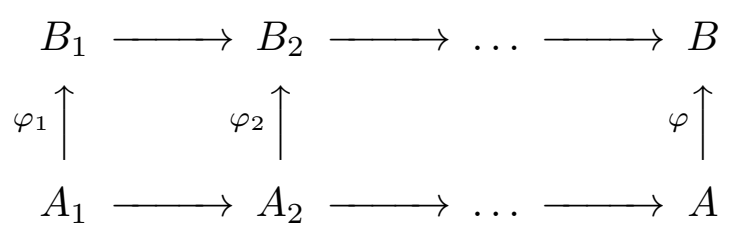

with the isomorphism $\varphi$ being obtained as the pointwise limit of the $\varphi_{i}$ taken in a suitable sense. More precisely,

$$
\varphi p_{n}(a)=\lim _{m \rightarrow \infty} q_{m} \varphi_{m} p_{m, n}(a)
$$

for all $a \in A_{n}$. Therefore

$$
\Gamma\left(\left[\varphi p_{n}\right]\right)(x)=\lim _{m \rightarrow \infty} \Gamma\left(\left[q_{m}\right]\right) \Gamma\left(\left[\varphi_{m}\right]\right) \Gamma\left(\left[p_{m, n}\right]\right)(x)
$$

in the discrete topology, for all $x \in \underline{K}\left(A_{n}\right)$. Since $\Gamma\left(\left[\varphi_{m}\right]\right)=\Gamma\left(\alpha_{m}\right)$ and since the first diagram is commutative we see that

$$
\begin{gathered}
\Gamma([\varphi]) \Gamma\left(\left[p_{n}\right]\right)(x)=\lim _{m \rightarrow \infty} \Gamma\left(\left[q_{m}\right] \alpha_{m}\right) \Gamma\left(\left[p_{m, n}\right]\right)(x)= \\
\quad=\lim _{m \rightarrow \infty} \Gamma\left(\alpha\left[p_{m}\right]\right) \Gamma\left(\left[p_{m, n}\right]\right)(x)=\Gamma(\alpha) \Gamma\left(\left[p_{n}\right]\right)(x)
\end{gathered}
$$

for all $x \in \underline{K}\left(A_{n}\right)$. We conclude that $\Gamma([\varphi])=\Gamma(\alpha)=\phi$ since $\underline{K}(A)=\underline{\varliminf}\left(\underline{K}\left(A_{n}\right), \Gamma\left(\left[p_{m, n}\right]\right)\right)$.

Let $A$ and $B$ be as in Theorem 3.6. Suppose that $A$ and $B$ are unital and $p_{n}$, $q_{m}$ are unit-preserving. We show that two $*$-homomorphisms $\varphi, \psi: A \rightarrow B$ which induce the same map $\underline{K}(A) \rightarrow \underline{K}(B)$ are approximately unitarily equivalent. Using the semiprojectivity of the $A_{n}$, we find sequences of $*$-homomorphisms $\varphi_{n}, \psi_{n}$ : $A_{n} \rightarrow B_{n}$ yielding approximations for $\varphi$ and $\psi$ and such that $\left[\varphi_{n}\right]=\left[\psi_{n}\right]$ in

$$
K K\left(A_{n}, B\right)=\operatorname{Hom}_{\Lambda}\left(\underline{K}\left(A_{n}\right), \underline{K}(B)\right) .
$$

Then using the step four in the Theorem 7.1 of [Ell] one shows that for any finite subset $F \subset A_{n}$ and any $\epsilon>0$, there exist $m, r \geq n$ and a unitary $u \in U\left(A_{m}\right)$ such that

$$
\left\|u q_{m, r} \varphi_{m} p_{m, n}(a) u^{*}-q_{m, r} \psi_{m} p_{m, n}(a)\right\|<\epsilon
$$


for all $a \in F$. Since

$$
\begin{aligned}
& \varphi p_{n}(a)=\lim _{m \rightarrow \infty} q_{m} \varphi_{m} p_{m, n}(a), \\
& \psi p_{n}(a)=\lim _{m \rightarrow \infty} q_{m} \psi_{m} p_{m, n}(a),
\end{aligned}
$$

we conclude that $\varphi$ is approximately unitarily equivalent to $\psi$.

Conversely, if $\varphi$ is approximately unitarily equivalent to $\psi$, then by using the semiprojectivity of $\mathbb{I}_{n}, C_{0}(0,1)$ and $\mathbb{C}$, it follows that the two $*$-homomorphisms induce the same map $\underline{K}(A) \rightarrow \underline{K}(B)$.

For a unital $\mathrm{C}^{*}$-algebra $A$ let $\overline{\operatorname{Inn}(A)}$ denote the group of approximately-inner automorphisms of $A$.

Corollary 3.7. Let $A$ be a unital AD algebra of real rank zero. Then there is a short exact sequence of groups

$$
1 \rightarrow \overline{\operatorname{Inn}(A)} \rightarrow \operatorname{Aut}(A) \rightarrow \operatorname{Aut}_{\Lambda}(\underline{K}(A),[1])^{+} \rightarrow 1 .
$$

This exact sequence is interesting even for simple AD algebras, as we are going to explain in a moment. We need a preliminary discussion. Following Rørdam [Rø], we let $K L(A, B)$ and $\operatorname{ext}\left(K_{*}(A), K_{*}(A)\right)$ denote the quotient of $K K(A, B)$ and respectively $\operatorname{Ext}\left(K_{*}(A), K_{*}(A)\right)$ by $\operatorname{Pext}\left(K_{*}(A), K_{*}(A)\right)$. Using the UCT and the UMCT we see that the map $\Gamma$ induces an isomorphism

$$
\hat{\Gamma}: K L(A, B) \rightarrow \operatorname{Hom}_{\Lambda}(\underline{K}(A), \underline{K}(B)) .
$$

If $A=B$ this is an isomorphism of rings. It follows that we can identify $A u t_{\Lambda}(\underline{K}(A))$ with the group of units of the ring $K L(A, A)$. The ring structure of $K L(A, A)$ can be described using [RS, Thm. 7.10].

$$
K L(A, A) \cong \operatorname{Hom}\left(K_{*}(A), K_{*}(A)\right) \oplus \operatorname{ext}\left(K_{*}(A), K_{*}(A)\right) .
$$

The multiplication is induced by the usual action of Hom on Ext which passes to an action of Hom on ext since the pullback and the pushout of a pure extension are pure. The product of any two elements in ext is zero. For more details the reader is refered to $[\mathrm{RS}],[\mathrm{R} \varnothing]$, [Sc3]. It follows that

$$
\operatorname{Aut}_{\Lambda}(\underline{K}(A)) \cong \operatorname{Aut}\left(K_{*}(A)\right) \times \operatorname{ext}\left(K_{*}(A), K_{*}(A)\right) .
$$

Now suppose that $A$ is a simple, unital, infinite-dimensional AD algebra of real rank zero. Then $\operatorname{ext}\left(K_{0}(A), K_{1}(A)\right)=0$ since $K_{0}(A)$ is an inductive limit of finitely generated free abelian groups [Sc3]. An element $x \in \underline{K}(A)$ is positive if and only if it is zero or its $K_{0}$ component is positive [DL3]. Therefore it is easily seen that there is a bijection

$$
\operatorname{Aut}_{\Lambda}(\underline{K}(A),[1])^{+} \cong \operatorname{Aut}\left(K_{0}(A),[1]\right)^{+} \times \operatorname{Aut}\left(K_{1}(A)\right) \times \operatorname{ext}\left(K_{1}(A), K_{0}(A)\right) .
$$

The product of two elements is given by:

$$
\left(\alpha^{0}, \alpha^{1}, x\right) \circ\left(\beta^{0}, \beta^{1}, y\right)=\left(\alpha^{0} \beta^{0}, \alpha^{1} \beta^{1},\left(\alpha^{0}\right)_{*}(y)+\left(\beta^{1}\right)^{*}(x)\right) .
$$


If for instance $A$ is a simple real rank zero $\mathrm{AD}$ algebra with $K_{0}(A)=\mathbb{Z}[1 / 3]$, $K_{0}(A)^{+}=\mathbb{Z}[1 / 3]^{+}$, and $K_{1}(A)=\mathbb{Z} / 2$, then $\operatorname{Aut}\left(K_{*}(A),[1]\right)^{+}=\{1\}$ and

$$
\operatorname{ext}\left(K_{1}(A), K_{0}(A)\right) \cong K_{0}(A) / 2 K_{0}(A) \cong \mathbb{Z} / 2,
$$

hence

$$
\operatorname{Aut}_{\Lambda}(\underline{K}(A),[1])^{+} \cong \mathbb{Z} / 2 .
$$

In particular there exists an automorphism of $A$ that acts identically on K-theory, but whose KK class is not equal to the class of $i d_{A}$.

3.8. Corollary The classification Theorem 3.6 and Corollary 3.7 are true for $\mathrm{C}^{*}$-algebras of real rank zero which are inductive limits of $\mathrm{C}^{*}$-algebras of the form

$$
A_{n}=\bigoplus_{i=1}^{m} M_{k(i)}\left(C\left(X_{i}\right)\right)
$$

where $X_{i}$ are finite CW complexes with $K^{0}\left(X_{i}\right)$ torsion free and the dimensions of the spectra of $A_{n}$ form a bounded sequence.

Proof. Let $A$ be a $\mathrm{C}^{*}$-algebra as in the statement of the Corollary. By Theorem 2.4 in $[\mathrm{D}], A$ is isomorphic to an AD algebra.

\section{REFERENCES}

[B11] B. Blackadar, K-theory for Operator Algebras, M. S. R. I. Monographs No. 5, SpringerVerlag, Berlin and New York, 1986.

[B] William Browder, Algebraic K-theory with coefficients $\mathbb{Z} / p$, Geometric applications of homotopy theory I (M. G. Barratt and M. E. Mahowald, eds.), Lecture Notes in Mathematics 657, Springer-Verlag, Berlin Heidelberg New York, 1978, pp. 40-84.

[Br1] L. G. Brown, Extensions and the structure of $C^{*}$-algebras, Istituto Nazionale di Alta Mathematica, Symposia Mathematica 20 (1976), 539-566.

[Br2] L. G. Brown, The Universal coefficient theorem for Ext and quasidiagonality, Operator Algebras and Group Representations, vol. 17, Pitman Press, Boston, London and Melbourne, 1983, pp. 60-64.

[BrD] L. G. Brown and M. Dadarlat, Extensions of $C^{*}$-algebras and quasidiagonality, to appear, J. of London Math. Soc..

[BMCS] R. R. Bruner, J. P. May, J. E. McClure and M. Steinberger, $H_{\infty}$ ring spectra and their applications, Lecture Notes in Mathematics 1176, Springer-Verlag, Berlin Heidelberg New York, 1986.

[Cu] J. Cuntz, K-Theory for certain $C^{*}$-algebras II, J. Operator Theory 5 (1981), 101-108.

[D] M Dadarlat, Approximately unitarily equivalent morphisms and inductive limit $C^{*}$ algebras, K-theory 9 (1995), 117-137.

[DL1] M. Dadarlat and T. A. Loring, K-homology, asymptotic representations and unsuspended E-theory, J. Funct. Anal. 126 (1994), 367-383.

[DL2] M. Dadarlat and T. A. Loring, Extensions of certain real rank zero $C^{*}$-algebras, Ann. Inst. Fourier 44 (1994), 907-925.

[DL3] M. Dadarlat and T. A. Loring, Classifying $C^{*}$-algebras via ordered mod-p K-theory, Preprint 1994.

[Ei] S. Eilers, A complete invariant for AD algebras with bounded torsion in $K_{1}$, preprint, 1994.

[F] L. Fuchs, Infinite abelian groups, Academic Press, New York and London.

[Ell] G. Elliott, On the classification of $C^{*}$-algebras of real rank zero I, J. reine angew. Math. 443 (1993), 179-219. 
[KS] J. Kaminker and C. Schochet, K-Theory and Steenrod homology: applications to the Brown-Douglass-Fillmore theory of operator algebras, Trans. Amer. Math. Soc. 227 (1977), 63-107.

[Kar] M. Karoubi, K-théorie algébrique de certaines algebres d'opèrateurs, Algèbres d'Opérateurs (P. de la Harpe, ed.), Lecture Notes in Mathematics 725, Springer-Verlag, Berlin Heidelberg New York, 1979, pp. 254-289.

[Kas] G. G. Kasparov, The operator K-functor and extensions of $C^{*}$-algebras, Math. URSS Izv. 16 (1981), 513-572.

[Lo1] T. A. Loring, C*-algebras generated by stable relations, J. Funct. Anal. 112 (1993), 159-201.

[Lo2] T. A. Loring, Stable relations II: corona semiprojectivity and dimension-drop $C^{*}$-algebras, to appear, Pacific J. Math..

[LP] T. A. Loring and G. K. Pedersen, Smoothing techniques in $C^{*}$-algebra theory, preprint, 1994.

[PS] V. Paulsen and N. Salinas, Two examples of non-trivial essentially n-normal operators, Indiana Univ. Math. J. 28 (1979), 711-724.

[Rø] M. Rørdam, Classification of certain infinite simple $C^{*}$-algebras, preprint 1993.

[RS] J. Rosenberg and C. Schochet, The Kunneth theorem and the universal coefficient theorem for Kasparov's generalized functor, Duke Math. J. 55 (1987), 431-474.

[Sa] N. Salinas, Relative quasidiagonality and KK-theory, Houston J. Math. 18 (1992), 97116.

[Sc1] C. Schochet, Topological methods for $C^{*}$-algebras III: axiomatic homology, Pacific J. Math. 114 (1984), 399-445.

[Sc2] C. Schochet, Topological methods for $C^{*}$-algebras IV: $\bmod p$ homology, Pacific J. Math. 114 (1984), 447-468.

[Sc3] C. Schochet, The UCT and the Milnor sequence for the Kasparov groups, Preprint.

[Sc4] C. Schochet, On the fine structure of the Kasparov groups, Preprint.

Department of Mathematics, Purdue University, West lafayette, IN 47907 USA

Department of Mathematics and Statistics, University of New Mexico, AlbuQUERQUE NM 87131 USA 